\section{Des cils battent dans le cerveau à un tempo finement régulé}

Grégory Conductier ${ }^{1}$, Angèle Viola ${ }^{2}$, Arnaud le Troter ${ }^{2}$, Jean-Louis Nahon ${ }^{1 *}$, Alice Guyon ${ }^{1 *}$
${ }^{1}$ Institut de pharmacologie moléculaire et cellulaire (IPMC), UMR 7275 CNRS, Université Nice Sophia Antipolis,

660 , route des lucioles, 06560 Valbonne, France.

${ }^{2}$ Centre de résonance magnétique biologique et médicale (CRMBM), UMR 7339 CNRS, Faculté de médecine la Timone, 27, boulevard Jean Moulin, 13005 Marseille, France.

${ }^{\star}$ Ces deux auteurs ont codirigé l'étude. gregory.conductier@ipmc.cnrs.fr nahonjl@ipmc.cnrs.fr alice.guyon@ipmc.cnrs.fr
Flux du liquide céphalo-rachidien et cils épendymaires

Le système nerveux central (regroupant encéphale et moelle épinière) n'est pas en contact direct avec les os qui l'entourent (boîte crânienne et vertèbres, respectivement). Il est comme maintenu en suspension dans un liquide appelé liquide céphalorachidien (LCR). Ce liquide est synthétisé par filtrage du sang artériel au niveau de structures spécialisées, les plexus choroïdes, à l'intérieur même du cerveau. En effet, le cerveau contient des cavités appelées ventricules, au sein desquelles le LCR circule d'avant en arrière. Ce déplacement est illustré pour le cerveau humain dans la Figure $1 A$. Chez les mammifères (homme, Figure IA ; souris, Figure $1 B)$, il existe quatre ventricules distincts. Dans le sens de sa circulation, le LCR, produit dans les plexus choroïdes, traverse d'abord deux grands ventricules latéraux (VL, un par hémisphère cérébral) avant de rejoindre le $3^{e}$ ventricule ( $3 \mathrm{~V}$, composé d'une partie dorsale et d'une partie ventrale). Ces ventricules convergent pour former l'aqueduc de Sylvius qui se déverse dans le $4^{\mathrm{e}}$ ventricule (4V). À ce niveau, le LCR peut sortir du système nerveux central et rejoindre un espace localisé entre les méninges (espace sous dural) ou emprunter le canal central de la moelle épinière (canal de l'épendyme). À son extrémité, il remonte dans l'espace sous dural tout le long de la colonne vertébrale et rejoint alors le flux sortant directement du cerveau. Le LCR est alors, soit réabsorbé par les plexus, soit éliminé au niveau des sinus veineux au sommet du crâne et dans la circulation lymphatique au niveau des bulbes olfactifs. Chez l'homme, le renouvellement total du volume de LCR est réalisé en huit heures environ.

À l'intérieur du système ventriculaire, le flux principal de LCR est généré par les contractions du système cardiovasculaire, tandis que la circulation laminaire proche des parois serait favorisée par le battement rythmique de millions de cils qui tapissent les ventricules. Ces cils sont portés par des cellules épithéliales appelées épendymocytes. Dans notre corps, des cils motiles semblables tapissent également les poumons et l'oviducte. Chacune de ces cellules possède à sa surface quelques dizaines de cils. Leur battement se fait en rythme à une fréquence très élevée, de l'ordre de la dizaine de mouvements par seconde. Afin de permettre la synchronisation globale du flux en bordure des ventricules cérébraux, plusieurs cellules voisines sont couplées entre elles par des jonctions communicantes (gap junctions) qui permettent la diffusion de différentes molécules, comme les ions calcium, capables de moduler la fréquence de battement des cils [1, 2].

Le LCR a longtemps été considéré comme un moyen de protéger le tissu nerveux, mou et fragile, de contraintes mécaniques violentes, et de maintenir une pression intracrânienne constante. Cependant, ses fonctions sont bien plus nombreuses: elles incluent le transport de nutriments et de molécules informatives (neuromodulateurs, hormones) vers le cerveau, l'élimination des déchets ou encore la migration de cellules comme les progéniteurs neuronaux [3]. Ainsi, les battements ciliaires, en favorisant cette circulation, pourraient participer au maintien de l'homéostasie cérébrale et à la transmission à distance d'informations de manière diffuse dans le LCR. D'un point de vue pathologique, tout dysfonctionnement dans le développement des cils épendymaires peut conduire à une accumulation, parfois mortelle, du LCR ou hydrocéphalie [4, 5]. L'accumulation de LCR dans le cerveau pourrait être liée au rôle déterminant des battements ciliaires dans les zones étroites du cerveau, comme l'aqueduc de Sylvius ou la base du 3V, mais cela n'a encore jamais été démontré.

Grâce à des études récentes, les mécanismes contrôlant la mise en place

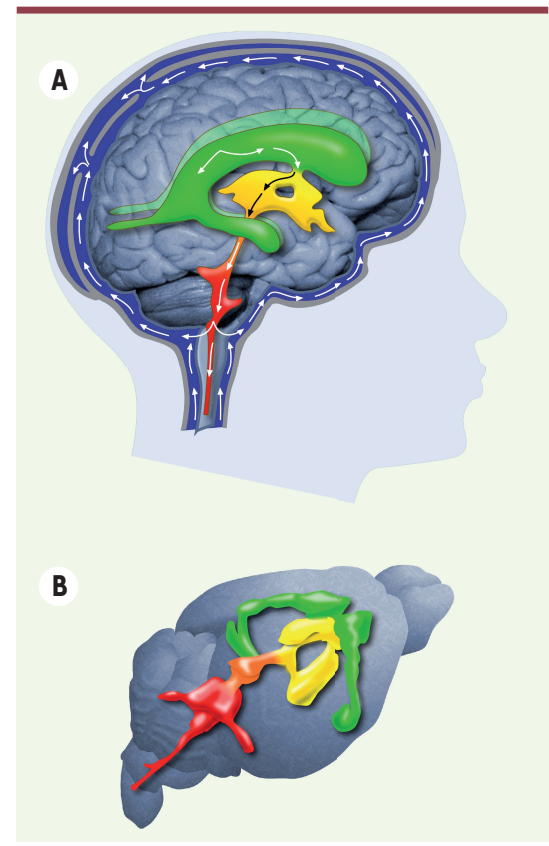

Figure 1. Circulation du liquide céphalo-rachidien dans les cavités ou ventricules du cerveau chez l'homme (A) et la souris (B). 


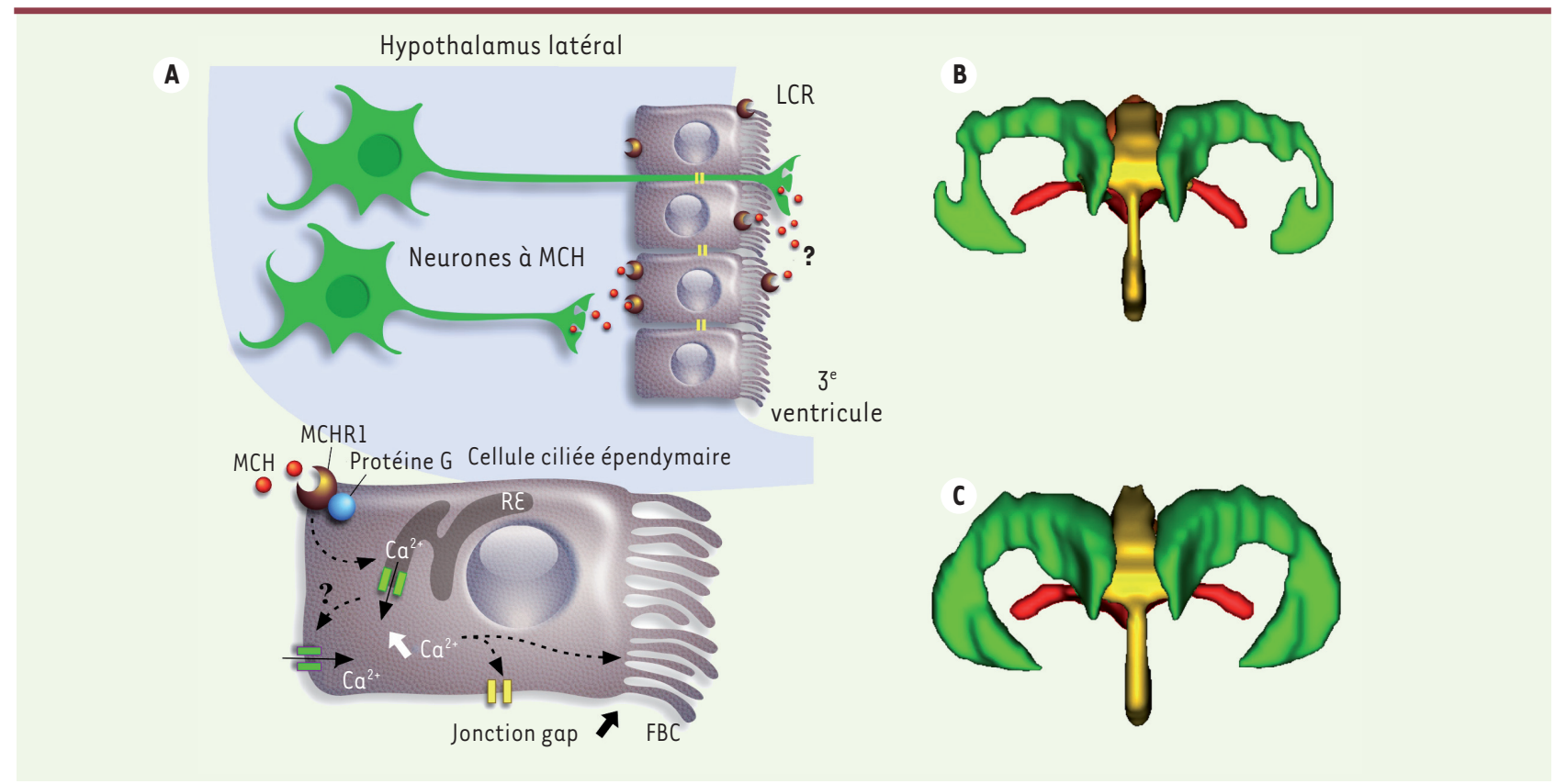

Figure 2. Rôle de la $\mathrm{MCH}$ (melanin concentrating hormone) dans la régulation de la $\mathrm{FBC}$ (fréquence des battements des cils) et du volume des ventricules. A. Schéma montrant les mécanismes d'action par lesquels la MCH peut moduler la fréquence de battement des cils des épendymocytes dans la partie ventrale du $3^{e}$ ventricule. La MCH libérée à la base des épendymocytes ou dans le LCR, en se liant à ses récepteurs MCHRl, induit une élévation de calcium entraînant une augmentation de la fréquence de battements des cils. B. Représentation en $3 D$ en vue frontale des ventricules cérébraux d'une souris sauvage, reconstruite à partir d'images obtenues en imagerie par résonance magnétique (IRM). C. Représentation en 3D en vue frontale des ventricules cérébraux d'une souris KO MCHRl, reconstruite à partir d'images obtenues en imagerie par résonance magnétique (IRM). $R \varepsilon$ : réticulum endoplasmique.

ou le fonctionnement des cils commencent à être connus. Par exemple, les mécanismes régissant l'orientation des cils [6] ou la synchronisation de leurs battements au sein d'une cellule viennent d'être élucidés [7]. Cependant, les mécanismes physiologiques qui contrôlent la fréquence de battement des cils étaient jusqu'à présent peu explorés. Dans une étude publiée dans Nature Neuroscience [8], nous nous sommes intéressés à ce problème en étudiant les battements ciliaires des cellules épendymaires bordant les ventricules cérébraux chez la souris, en collaboration avec des équipes françaises, belges et canadiennes.

\section{L'hormone de mélano-concentration module la fréquence de battement} des cils bordant le troisième ventricule cérébral

Notre équipe travaille depuis de nombreuses années sur l'hormone de mélano-concentration $(\mathrm{MCH})$, un neuromodulateur peptidique produit sélectivement par certains neurones localisés au niveau de l'aire latérale de l'hypothalamus, et principalement impliqués dans la régulation de la prise alimentaire, du métabolisme, du sommeil et du stress chez les mammifères $[9,10]$. Afin de visualiser facilement ces neurones dans des coupes de cerveau de souris, les neurones à MCH ont été rendus fluorescents. Nous avons ainsi constaté, dans les coupes de cerveau maintenues en survie, que les prolongements des neurones à $\mathrm{MCH}$ entrent en contact spécifiquement avec certains épendymocytes dans la partie ventrale du $3 \mathrm{~V}$, mais pas avec ceux des VL ou du 4V. Nous avons donc souhaité savoir si les neurones à $\mathrm{MCH}$ pouvaient contrôler l'activité des cellules ciliées. Chez la souris, la MCH module les fonctions cellulaires en se liant à des récepteurs membranaires spécifiques (MCHRl) qui activent, à la face interne de la membrane, des protéines $G$ capables d'induire l'activation de voies de signalisation intracellulaires ${ }^{1}$. Nos expériences, réalisées in vitro, ont d'abord révélé l'expression du MCHRl à la membrane des épendymocytes (Figure 2A). De façon spectaculaire, seules les cellules ciliées en contact avec les fibres à $\mathrm{MCH}$ expriment le MCHRI dans le $3^{e}$ ventricule. L'application de MCH sur les épendymocytes bordant le $3^{\mathrm{e}}$ ventricule, en stimulant le MCHRl, induit dans ces cellules une élévation des concentrations de calcium intracellulaire (Figure 2A). Nous avons alors émis l'hypothèse que la MCH libérée à proximité de ces cellules pourrait en moduler la fréquence de battements de cils, à l'instar d'autres composés dans d'autres systèmes, comme l'ATP, la

\footnotetext{
1 Voir le numéro de médecine/sciences consacré aux récepteurs couplés aux protéines $G, m / s n^{\circ} 10$, vol. 28 , octobre 2012.
} 
sérotonine ou le PACAP (pituitary adenylate cyclase-activating polypeptide). Pour démontrer cette hypothèse, nous avons mis au point un système d'enregistrement et de mesure de la fréquence de battements de cils (FBC) sur des tranches de cerveau ${ }^{2}$ et montré que l'application de $\mathrm{MCH}$ augmente cette fréquence dans les cellules bordant le $3 \mathrm{~V}$ ventral. Aucune modulation n'est observée dans les autres ventricules, ni même dans la partie la plus dorsale du $3^{e}$ ventricule. Cette augmentation de la fréquence de battements de cils est aussi observée en réponse à la stimulation à distance des corps cellulaires des neurones à $\mathrm{MCH}$, qu'elle soit électrique, chimique ou optogénétique. L'ensemble de ces effets est inhibé par l'action d'un antagoniste spécifique du MCHRl. Fait remarquable, l'invalidation du gène mchrl provoque une réduction de plus de $50 \%$ de la fréquence de battements des cils en l'absence de toute forme de stimulation et, bien évidemment, elle abolit l'effet activateur de la MCH sur la fréquence de battements des cils.

Chez ces mêmes animaux, des études in vivo en imagerie par résonance magnétique montrent un élargissement notable du $3 \mathrm{~V}$ et des VL, ainsi qu'un rétrécissement de l'aqueduc de Sylvius (Figure 2B-C). Nous avons donc émis l'hypothèse que la diminution locale de la fréquence de battements des cils dans le $3 \mathrm{~V}$ produit un ralentissement de l'écoulement du LCR induisant son accu-

2 Pour plus d'informations, consulter http://hal.archivesouvertes.fr/hal-00806681 mulation en amont du $3 \mathrm{~V}$ et une diminution de volume en aval de ce ventricule.

\section{Conclusions et perspectives}

La fréquence de battement des cils bordant le $3^{e}$ ventricule est étroitement contrôlée par l'activité des neurones de l'hypothalamus produisant la MCH (Figure $2 \mathrm{~A}$ ), et un dysfonctionnement de ce contrôle peut entraîner un élargissement notable des ventricules (Figure 2B-C). Nos résultats appellent donc à la prudence dans l'utilisation d'antagonistes de la MCH qui sont envisagés, dans le traitement de l'obésité en particulier. Ces résultats majeurs en termes physiopathologiques ouvrent une nouvelle piste dans la thérapie des hydrocéphalies chez l'homme par l'action de substances pharmacologiques modulant la fréquence des battements ciliaires. Ils illustrent également toute la complexité des modes d'action des neuropeptides dans le cerveau, ces derniers pouvant à la fois activer des populations neuronales via la transmission synaptique rapide, et moduler l'activité cérébrale globale à une échelle de temps plus lente en régulant les flux ventriculaires. $\diamond$

Beating frequency of motile cilia lining the third cerebral ventricle is finely tuned by the hypothalamic peptide $\mathrm{MCH}$

\section{REMERCIEMENTS}

Ces travaux ont été réalisés en collaboration avec le centre de résonnance magnétique biologique et médicale (CNRS, université Aix-Marseille), le laboratoire d'infor- matique, signaux et systèmes de Sophia Antipolis (I3S, CNRS, université Nice SophiaAntipolis), le centre de recherche Jean-Pierre Aubert (Inserm, université Lille-2), l'université de Franche-Comté, l'université de Liège (Belgique) et l'Institut universitaire en santé mentale Douglas (Canada). Nous remercions Franck Aguila pour son travail artistique sur les figures.

\section{LIENS D'INTÉRÊT}

Les auteurs déclarent n'avoir aucun lien d'intérêt concernant les données publiées dans cet article.

\section{RÉFÉRENCES}

1. Zhang L, Sanderson MJ. Oscillations in ciliary beat frequency and intracellular calcium concentration in rabbit tracheal epithelial cells induced by ATP.J Physiol 2003 ; 546 : 733-49.

2. Prochnow N, Dermietzel R. Connexons and cell adhesion: a romantic phase. Histochem Cell Biol $2008 ; 130: 71-7$.

3. Veening JG, Barendregt HP. The regulation of brain states by neuroactive substances distributed via the cerebrospinal fluid; a review. Cerebrospinal Fluid Res $2010 ; 7: 1$.

4. Tissir F, Qu Y, Montcouquiol M, et al. Lack of cadherins Celsr2 and Celsr3 impairs ependymal ciliogenesis, leading to fatal hydrocephalus. Nat Neurosci 2010 ; $13: 700-7$.

5. Wilson GR, Wang HX, Egan GF, et al. Deletion of the Parkin co-regulated gene causes defects in ependymal ciliary motility and hydrocephalus in the quakingviable mutant mouse. Hum Mol Genet 2010 ; 19 : 1593-1602.

6. Mirzadeh Z, Han YG, Soriano-Navarro M, et al. Cilia organize ependymal planar polarity. J Neurosci 2010 ; $30: 2600-10$.

7. Kunimoto K, Yamazaki Y, Nishida T, et al. Coordinated ciliary beating requires $0 \mathrm{df} 2$-mediated polarization of basal bodies via basal feet. Cell 2012 ; 148 : 189-200.

8. Conductier G, Brau F, Viola A, et al. Melaninconcentrating hormone regulates beat frequency of ependymal cilia and ventricular volume. Nat Neurosci $2013 ; 16: 845-7$

9. Bittencourt JC, Presse F, Arias C, et al. The melaninconcentrating hormone system of the rat brain: an immuno- and hybridization histochemical characterization. J Comp Neurol 1992 ; 319 : 218-45.

10. Presse F, Nahon JL. MCH. In : Kastin A, ed. Handbook of biologically active peptide. 2013 : 828-37.

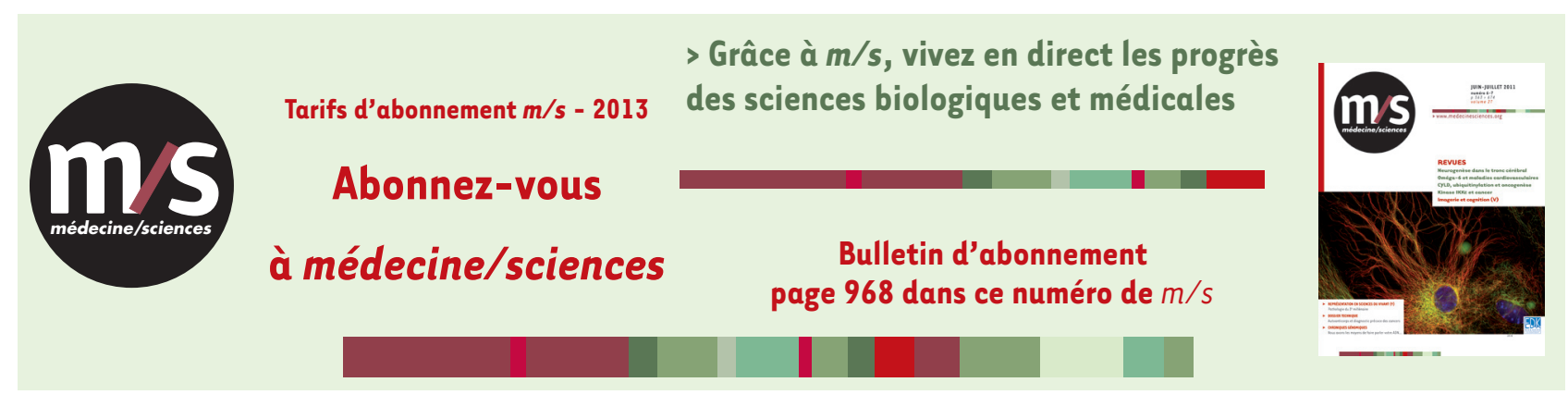

